\title{
Contribution à la création d'une base de données spatiale pour la gestion des forages en milieu fissuré: cas du Denguelé (Nord-Ouest de la Côte d 'Ivoire)
}

\author{
Arthur Brice KONAN-WAIDHET ${ }^{1 *}$, Konan Emmanuel KOUADIO ${ }^{2}$, Brou DIBI ${ }^{3}$, \\ Gabriel Etienne $\mathrm{AKE}^{4}$ et Issiaka SAVANE ${ }^{5}$ \\ ${ }^{1}$ Laboratoire Géosciences et Environnement, Université d'Abobo-Adjamé, Abidjan (Côte d'Ivoire), 02 BP 801 \\ Abidjan 02.(225)05747336.E-mail: konanwab@yahoo.fr \\ ${ }^{2}$ Sciences et Techniques de l'Eau et de l'Environnement, UFR des Sciences de la Terre et des Ressources \\ Minières, Université de Cocody, Abidjan (Côte d'Ivoire). 22 BP 582 Abidjan 22. (225) 05611839, \\ E-mail:emmakouadio@hotmail.com \\ ${ }^{3,5}$ Laboratoire Géosciences et Environnement, Université d'Abobo-Adjamé, Abidjan (Côte d'Ivoire), 02 BP \\ 801 Abidjan 02. (225)03313031.E-mail: dibrou2003@yahoo.fr \\ ${ }^{4}$ Laboratoire Sciences et Techniques de l'Eau et de l'Environnement, UFR des Sciences de la Terre et des \\ Ressources Minières, Université de Cocody, Abidjan (Côte d'Ivoire). 22 BP 582 Abidjan 22. (225) 07734726, \\ E-mail:ak_gabe@yahoo.fr \\ ${ }^{5}$ Laboratoire Géosciences et Environnement, Université d'Abobo-Adjamé, Abidjan (Côte d'Ivoire), 02 BP 801 \\ Abidjan 02. (225)07773422, E-mail: savanei@hotmail.com \\ *Auteur correspondant, E-mail: konanwab@yahoo.fr, Tel : (225)05747336.
}

\section{RESUME}

Le choix du site d'un forage productif constitue une préoccupation réelle pour les gestionnaires de la région du Denguelé, à cause de l'inorganisation et de la mauvaise exploitation des données disponibles. La structuration de ces données en vue de capitaliser l'ensemble de ces informations est nécessaire pour aider à la prise de décision. L'objectif de ce travail est de concevoir une base de données spatiale pour aider aux choix des sites de forage à travers des traitements possibles des données. La conception de cette base de données a été faite selon la méthode MERISE qui se caractérise par trois niveaux : conceptuel, logique et physique. Le résultat du travail est un outil de démonstration qui permet de faire la saisie, la mise à jour et offre la possibilité d'interroger la base de données. Ainsi, ce Système d'Information à Référence Spatiale (SIRS), enrichi par une multitude de requêtes peut constituer pour les décideurs un tableau de bord pour le choix du site des forages de manière rationnelle.

(C) 2010 International Formulae Group. All rights reserved.

Mots clés : Gestion, Forage, SIRS, Merise, Denguélé, Côte d'Ivoire.

\section{INTRODUCTION}

L'approvisionnement en eau potable est l'un des objectifs majeurs des projets de développement à travers le monde. En Côte d'Ivoire, malgré de nombreux efforts consentis depuis 1973, des difficultés demeurent car l'eau potable n'est pas encore accessible à tous (Kouadio et al., 2008). Ces difficultés pourraient s'expliquer par une mauvaise exploitation et gestion des données disponibles. La région du Denguelé ne fait pas exception à la règle. 
Selon Böker (2003), il est opportun de trouver des solutions toujours plus efficaces pour explorer, chercher et gérer les ressources en eau. La recherche de solutions adéquates de valorisation et de gestion des forages, nécessite l'organisation de toutes les données nécessaires dans une base de données spatiale ou système d'information à référence spatiale (SIRS). Cet outil offrira alors la possibilité d'extraire rapidement les informations nécessaires et répondants clairement à des objectifs prédéfinis (Barazzuoli et al., 1999). Pour mener à bien cette étude, la méthode MERISE a été adoptée. Elle implique une structuration des données et permet de construire des systèmes durables et évolutifs. En effet, les SIRS sont considérés comme d'excellents outils d'analyse pour la planification et la gestion, compte tenu de leurs capacités de mémoriser, de manipuler et de produire de l'information spatiale (Lili Chabaane et al., 2002).

Il s'agit, dans le cadre de cette étude, d'élaborer une base de données spatiale pour aider aux choix des sites de forage à travers des traitements possibles des données. Ainsi, la conception et la réalisation d'un SIRS intégrant les informations sur les forages permettent de répondre aux besoins des gestionnaires, des investisseurs privés et des professionnels travaillant dans le domaine des ressources en eaux (Kedaid, 2006).

\section{MATERIEL ET METHODES}

\section{Présentation de la région du Denguelé}

La région du Denguelé est située au Nord-Ouest de la Côte d'Ivoire entre les longitudes $7^{\circ}$ et $8^{\circ} 15^{\prime} \mathrm{W}$ et les latitudes $9^{\circ}$ et $10^{\circ} 25^{\prime} \mathrm{N}$ (Figure 1). Elle présente une morphologie dominée par une pénéplaine incisée par quelques vallées. L'altitude des sommets varie entre 600 et $900 \mathrm{~m}$ (INS, 2004). On y note localement la présence de plateaux cuirassés. La géologie de cette région est variée avec la présence de roches métamorphiques (schistes, gneiss, métavolcanites, etc.) et roches magmatiques dominées par les granitoïdes (granite à biotite, granite à deux micas, granodiorite, etc.). Les altérites meubles ont une épaisseur très variable à l'échelle régionale $(0$ à $50 \mathrm{~m}) \mathrm{du}$ fait du degré variable d'érosion, des profils d'altération et de la grande variété pétrographique. La population est estimée à 222446 habitants (INS, 2000).

\section{Matériel}

Le matériel est constitué de données et de logiciels. La conception de ce SIRS a nécessité l'utilisation d'une carte topographique au 1/200 000, fournie par le Centre de Cartographie et de Télédétection (CCT) d'Abidjan, et des données descriptives de forages.

Plusieurs logiciels ont été utilisés. Ce sont: Microsoft Access 2003 pour le traitement des données descriptives, Power AMC 9.5 pour la modélisation des données et MapInfo 6.5 pour l'implantation des données spatiales et la mise en œuvre du prototype.

\section{Méthode de conception de la base de données}

La démarche adoptée pour concevoir la base de données est illustrée par la Figure 2. Les données collectées ont été organisées dans une base de données sur laquelle seront formulées des requêtes. Cette structuration des données ou modélisation des données est utile dans la réalisation d'un SIRS (Pennober et al., 2004).

\section{Modélisation conceptuelle des données (MCD)}

Le modèle conceptuel consiste à identifier et à décrire les objets et leurs relations. Pour Golay (1997), le modèle conceptuel ne correspond pas à une réalité absolue, mais plutôt à la réalité qui peut intéresser les utilisateurs et doit être le plus indépendant possible des technologies qui seront mises en œuvre et du logiciel de système d'information géographique qui sera utilisé. Ce modèle a été élaboré à partir du logiciel Power AMC 9.5 selon le formalisme entité-relation après de nombreuses « 
itérations ». Il nécessite de déterminer les entités, leurs attributs relatifs et les relations qui peuvent exister entre elles.

\section{Détermination des entités}

Toutes les entités ont été déterminées en respectant les critères de pertinence vis-àvis de la gestion des forages (Lakhoua, 2007). Leur description précise est une phase essentielle car elle permet de dissiper toutes formes d'équivoque sur les termes utilisés. Pour une meilleure compréhension du modèle, elles ont été réparties entre trois classes fondamentales à savoir: les objets cartographiques, les entités à référence spatiale et les entités sans dimension spatiale.

\section{Objets cartographiques}

Ils constituent un repère cartographique et sont intégrés dans le SIRS comme fond de plan ou habillage afin de mieux visualiser les aspects géographiques. Il s'agit de la carte topographique et de l'image satellitaire.

\section{Entités à référence spatiale}

Ce sont les éléments qui peuvent être déterminés par des coordonnées dans un repère géographique donné. Il s'agit de l'entité :

Forage: Il s'agit d'une entité qui constitue l'élément central du SIRS. A ces ouvrages sont rattachées des données descriptives. Le paramètre le plus important pour cette entité est la coordonnée géographique du forage. Sa position sur la surface du globe doit être prise avec le plus grand sérieux car elle permet la liaison avec le domaine spatial. Cette entité permet également d'identifier les localités qui ont bénéficié des forages lors des différentes campagnes réalisées au niveau de la région.

\section{Entités sans dimension spatiale ou descriptives}

Ce sont les éléments descriptifs du territoire qui sont liés aux entités spatiales par un lien physique ou relationnel. Il s'agit de:

Campagne: Il s'agit d'identifier le projet à l'origine des forages. C'est un élément qui permettra d'évaluer leur taux de succès.

\section{Sous préfecture}

Chaque campagne a lieu dans un village qui appartient à une sous préfecture. On pourra ainsi apprécier les zones qui bénéficient le plus souvent des forages, cela permettra de réparer les injustices faites à certaines localités.

Village

Toutes les données socio-économiques sont liées à l'entité village car toutes les activités s'organisent autour du village. De même, tout village administratif, quelle que soit sa population, doit être pourvu d'au moins un Point d'Eau Moderne (PEM). Une fiche descriptive pour chaque village permet d'avoir un premier aperçu de la taille de sa population (nombre d'homme, de femme, d'enfant) et d'identifier les activités économiques.

\section{Activité éducative}

Afin d'assurer la pérennité des ouvrages, il est primordial de consacrer une partie importante du temps à la formation et la sensibilisation. L'information, la communication et la formation sont des démarches indispensables pour établir des solutions efficaces à une gestion durable des forages. L'objectif principal de cette entité est d'aboutir à une prise de conscience collective en vue d'une gestion communautaire des ouvrages. Elle est rattachée à l'entité comité de gestion.

Géologie

Cette entité est rattachée à l'entité forage. Elle permet d'apprécier la nature des formations encaissantes. C'est une donnée nécessaire qui va intervenir dans l'analyse de la productivité des forages.

\section{Paramètres Chimiques}

Cette entité est rattachée à l'entité forage et permet d'apprécier la qualité des eaux souterraines de la région.

\section{Paramètres hydrodynamiques}

Cette entité regroupe toutes les données interprétées obtenues à partir des résultats des essais de pompage.

\section{Comité de gestion}

Cette entité est également rattachée à l'entité forage. On se propose de mettre en place un comité de gestion de l'ouvrage confié à un responsable choisi par le chef de village à 
qui un compte rendu est fait régulièrement sur l'état de l'ouvrage. Ce responsable se charge également de sensibiliser les ménages sur la nécessité d'entretenir l'ouvrage. En effet, l'état défectueux de certaines pompes amène les populations à la consommation des eaux de qualité douteuse (source naturelle, puits traditionnels et marigots) encore présentes dans un grand nombre de localités.

\section{Détermination des attributs et des relations}

Le choix des attributs relatifs à chaque entité repose également sur leur pertinence vis-à-vis des besoins en matière de gestion des forages.

Une relation est un lien entre deux entités ou une entité et une association. Toutes les relations ont été déterminées en respectant leurs pertinences vis-à-vis de la gestion des forages.

Afin d'intégrer l'ensemble des données dans la base de données, les modèles logique et physique ont été développés.

\section{Modélisation logique des données (MLD)}

Le MCD obtenu dans l'étape précédente a été traduit en modèle logique des données relationnelles (MLDR). Dans ce type de modèle, les entités et les associations sont représentées par des tableaux à deux dimensions, souvent appelés tables qui comprennent des tables primaires et secondaires (Golay, 1997). Le MLD organise donc les données du MCD dans des tables tout en permettant de garder les relations existantes.

\section{Mise en œuvre du prototype}

La mise en œuvre du prototype a consisté à implanter le modèle physique des données (MPD), c'est-à-dire la création des tables définies par le MLDR en y ajoutant les attributs correspondants, puis à effectuer des traitements réalisés afin de tester la qualité du modèle.

\section{Implantation des tables primaires}

On appelle "table primaire" toute table du modèle logique dont les objets peuvent être représentés graphiquement (Barbier, 2002). Ces tables primaires ont été créées sous MapInfo. Ces objets sont de type ponctuel (Forage) conformément au(x) pictogramme(s) indiqué(s) sur les entités du modèle conceptuel. En plus des références spatiales, ces tables contiennent également des données non spatiales. Pour le prototype, l'implantation de ces objets s'est effectuée en les digitalisant à partir d'un fond de carte topographique géoréférencée. Dans ces tables primaires, seul le code de l'objet est inscrit, le reste de la typologie se trouve dans les tables secondaires.

\section{Implantation des tables secondaires}

Les tables secondaires regroupent l'ensemble des tables qui n'ont pas de références spatiales et qui contiennent des informations sur le SIRS. Ces données sans dimension spatiale ont été traitées directement sous Microsoft Access pour la création et les mises à jour, puis elles ont été importées dans MapInfo. L'intégration des données provenant de Microsoft Access n'a posé aucun problème, puisque leur structure tabulaire permet un accès direct sous MapInfo par le lien ODBC (Open Data Base Connexion). Ainsi, les modifications apportées à la base de données entre deux sessions (par exemple, ajout d'un nouveau forage, suppressions) sont automatiquement prises en compte au niveau des tables.

\section{RESULTATS}

Modèle conceptuel des données (MCD)

Le modèle conceptuel des données (MCD) réalisé à partir des entités retenues apparaît, du point de vue graphique, comme un ensemble de polygones reliés par des polylignes (Figure 3). Il est constitué d'une série d'entités spatiales (symbolisées par un pictogramme) et non spatiales, connectées entre elles par des relations.

Le MCD permet ainsi de doter la base de données d'une structure solide qui permettra, d'une manière efficace, d'optimiser l'échange de données entre les applications. 
Modèle logique des données (MLD)

En tenant compte des différentes cardinalités, nous avons obtenu en définitive 08 tables descriptives et 01 table à référence spatiale. En effet, les liens entre les différentes entités, représentés par les relations dans le MCD, se sont transformés en attribut supplémentaire (relation de un à plusieurs) où l'identifiant devient la clé primaire.

\section{Exploitation du prototype}

L'exploitation du prototype consiste à interroger, à l'aide du langage d'interrogation SQL (Structured Query Language), la base de données géographique et d'en extraire des informations dont on a besoin (Lili-Chabaane et al., 2002). Ces informations sont de plusieurs formes, à savoir des cartes, des graphiques, des tableaux et des statistiques.

L'exploitation de ce prototype à travers trois exemples de requêtes permettra de localiser les forages qui ont un débit $\geq$ à 3 $\mathrm{m}^{3} / \mathrm{h}$ et dont l'épaisseur d'altérite est comprise entre 10 et $40 \mathrm{~m}$, les forages qui ont un débit $\geq$ à $3 \mathrm{~m}^{3} / \mathrm{h}$ et dont la profondeur totale est comprise entre 40 et $70 \mathrm{~m}$ et les formations géologiques dans lesquelles l'on rencontre des débits $\geq$ à $3 \mathrm{~m}^{3} / \mathrm{h}$. En effet, dans le cadre de l'hydraulique villageoise améliorée (HVA), le succès des forages est lié à un débit d'exploitation d'au moins $3 \mathrm{~m}^{3} / \mathrm{h}$.

Requête 1 : "Quels sont les forages qui ont un débit $\geq \grave{a} 3 \mathrm{~m}^{3} / \mathrm{h}$ et dont l'épaisseur d'altérite est comprise entre 10 et $40 \mathrm{~m}$ ? »

Cette requête renvoie au contenu de la colonne «nomvillage» de la table VILLAGE, le contenu de la colonne «épaisseur d'altérite » de la table FORAGE et le contenu de la colonne «débit» de la table PARA. HYDRODYNAMIQUE. La jointure entre les tables VILLAGE et FORAGE se fait sur « idvillage» dans la clause «Where» pour indiquer au système que la liaison entre les deux tables s'effectue sur cette colonne commune. Par contre, la jointure entre les tables FORAGE et PARA. HYDRODYNAMIQUE se fait sur «idforage » dans la clause «Where» pour indiquer au système que la liaison entre les deux tables s'effectue sur cette colonne commune. Les conditions posées concernent la valeur du débit qui est" $\geq 3 \mathrm{~m}^{3} / \mathrm{h}$ " et l'épaisseur d'altérite qui se situe entre "10 m" et "40 m" (Tableau 1).

Les réseaux hydrographique et routier sont utilisés comme toile de fond. Les forages identifiés à partir de cette requête sont ceux marqués d'un rectangle en surbrillance rouge (Figure 5) et sont localisés dans les villages (Tougousso, Massadougou, Odienne, Sirana, Kodougou, Zievasso, Sienso et Foula). L'analyse de la variation des débits en fonction de l'épaisseur montre qu'il n'y a pas une relation linéaire entre l'importance des débits et la puissance des altérites d'une manière générale.

Requête 2 : «Quels sont les forages qui ont un débit $\geq a ̀$ $3 \mathrm{~m}^{3} / \mathrm{h}$ et dont la profondeur totale est comprise entre 40 et $70 \mathrm{~m}$ ? »

Cette requête prend en compte trois éléments: le débit des forages, le nom des villages concernés par ces forages et la profondeur totale des forages. Le débit des forages est obtenu en joignant la table « Para. Hydrodynamique » et la table «Village». Cette opération permet d'avoir le nom des villages. Ce résultat est ensuite croisé avec la table «Géologie ». Les conditions posées concernent la valeur du débit qui est" $\geq 3$ $\mathrm{m}^{3} / \mathrm{h}$ " et dont la profondeur totale des ouvrages qui se situe entre " $40 \mathrm{~m}$ " et $70 \mathrm{~m}$ (Tableau 2).

L'extrait topographique est utilisé comme toile de fond dans cette requête. Les forages concernés sont indiqués par un rectangle en surbrillance rouge (Figure 6) sur l'extrait topographique et sont situés dans les localités suivantes: Tougousso, Massadougou, Odienne, Sirana, Zievasso, Sienso et Foula. Un graphe de répartition des débits en fonction de la profondeur totale de l'ouvrage a été établi (Figure 7).

Une analyse de ce graphique montre que les profondeurs comprises entre 40 et 70 $\mathrm{m}$ sont caractérisées par des débits plus importants pouvant atteindre $18 \mathrm{~m}^{3} / \mathrm{h}$ (Figure 
7). Au-delà d'une profondeur de $70 \mathrm{~m}$, on rencontre des forages à faibles débits. La tranche de profondeur optimale pour réaliser des forages économiques est comprise entre 40 et $70 \mathrm{~m}$. Ces résultats peuvent donc contribuer à orienter les prospections hydrogéologiques futures.

Requête 3 : Quelles sont les formations géologiques dans lesquelles l'on rencontre des débits $\geq \grave{a} 3 \mathrm{~m}^{3} / \mathrm{h}$ ? "

Cette requête prend en compte trois éléments: le débit des forages, le nom des villages concernés par ces forages et la nature de la roche encaissante. Le débit des forages est obtenu en joignant la table «Para. Hydrodynamique » et la table «Village».
Cette opération permet d'avoir le nom des villages qui est ensuite croisé avec la table «Géologie » en posant comme condition le choix du débit qui doit être $\geq 3 \mathrm{~m}^{3} / \mathrm{h}$ (Tableau $3)$. Les réseaux hydrographique et routier sont utilisés comme toile de fond. Les forages identifiés à partir de cette requête sont ceux marqués d'un rectangle rouge et se situent à Massadougou, Odienné, Sirana, Foula.

Les analyses portent sur les forages réalisés dans les granites à biotites et les gneiss. Les débits importants $\left(\mathrm{Q} \geq 3 \mathrm{~m}^{3} / \mathrm{h}\right)$ s'observent aussi bien dans les granites à biotite que dans les gneiss. Cependant, dans la majorité des cas, les débits d'exploitation des granites à biotite sont inférieurs à ceux des gneiss.

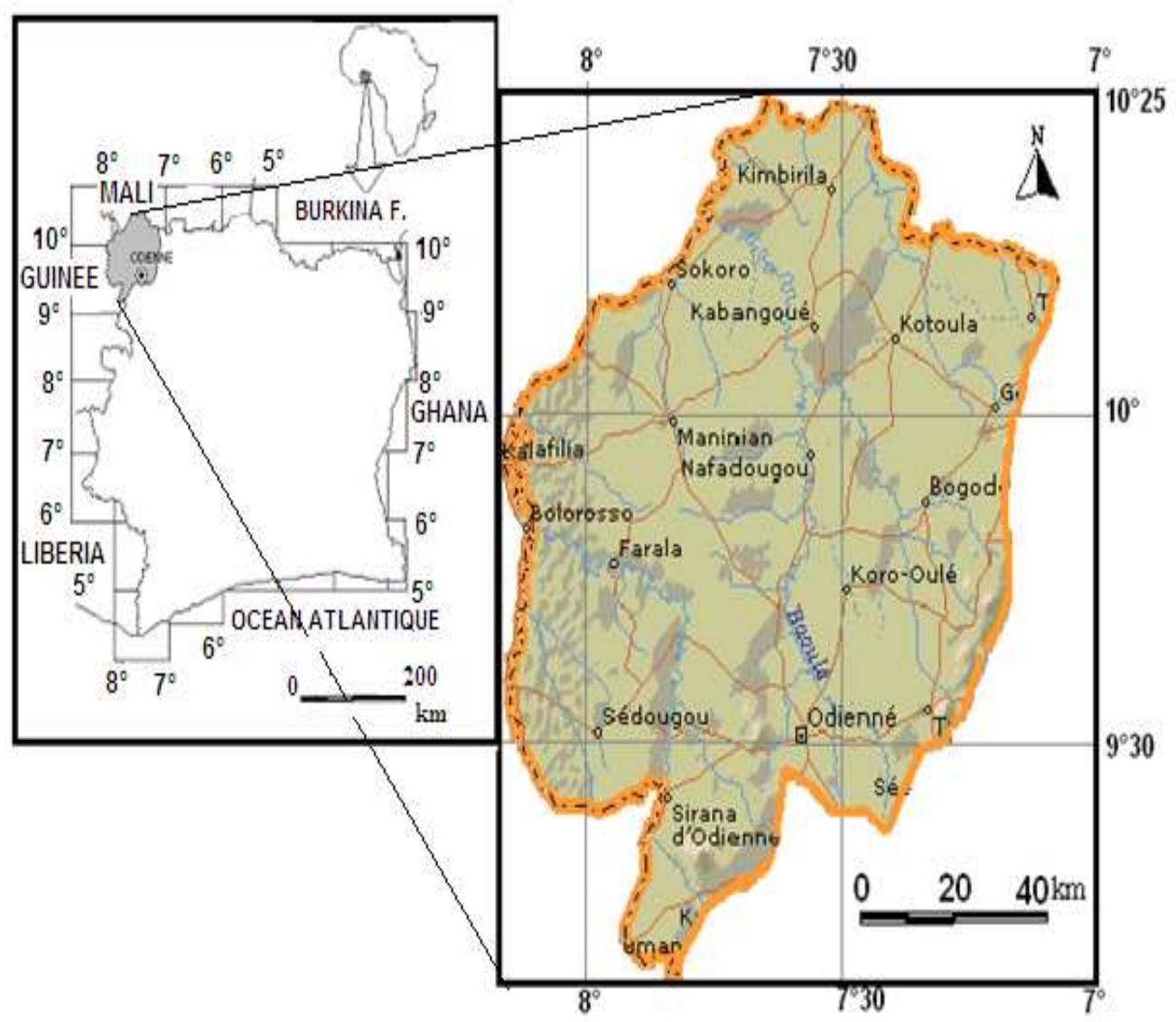

Figure 1 : Localisation de la région du Denguelé. 


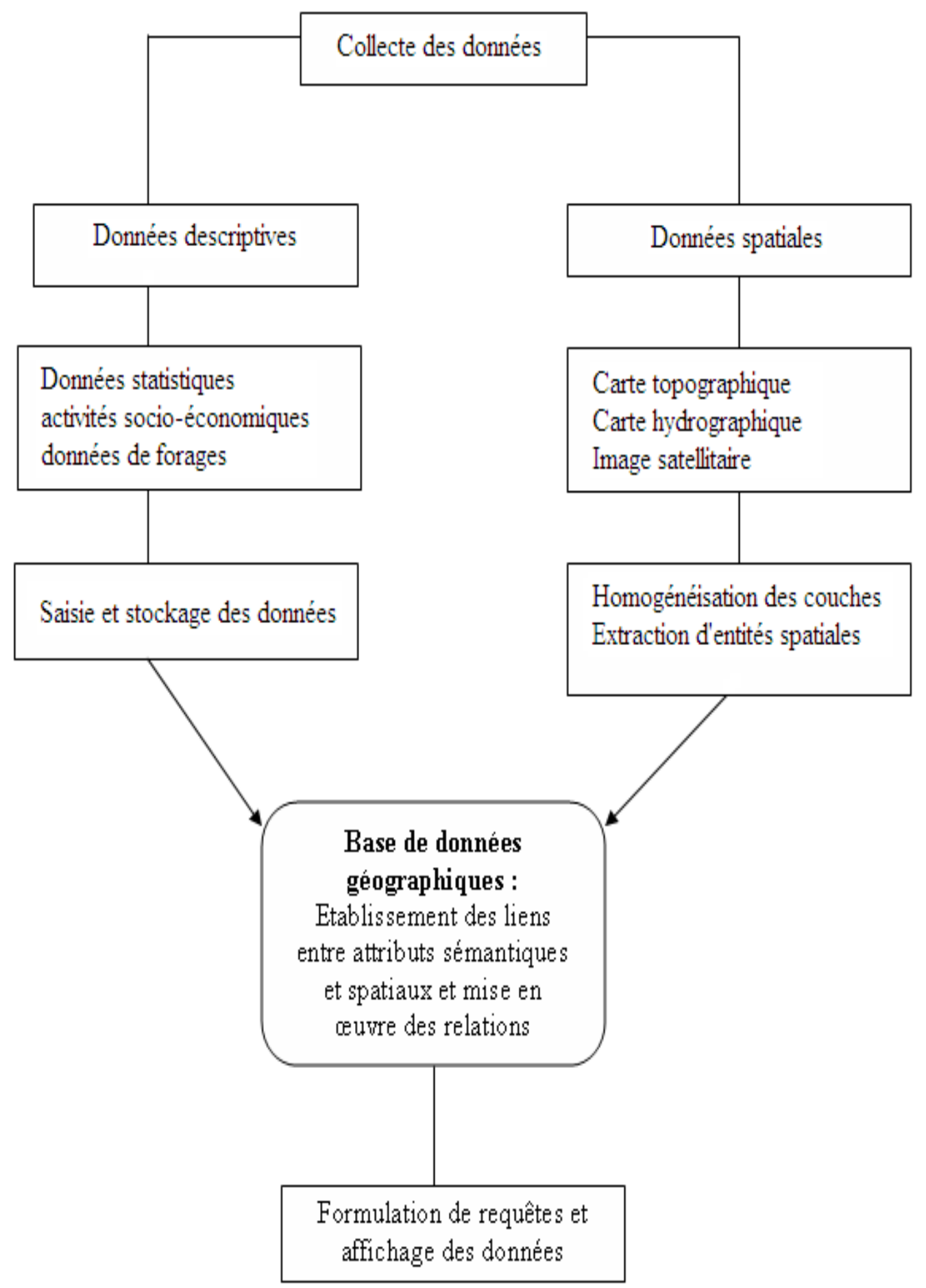

Figure 2: Organigramme conceptuel de la base de données. 
A. B. KONAN-WAIDHET et al. / Int. J. Biol. Chem. Sci. 4(6): 2046-2059, 2010

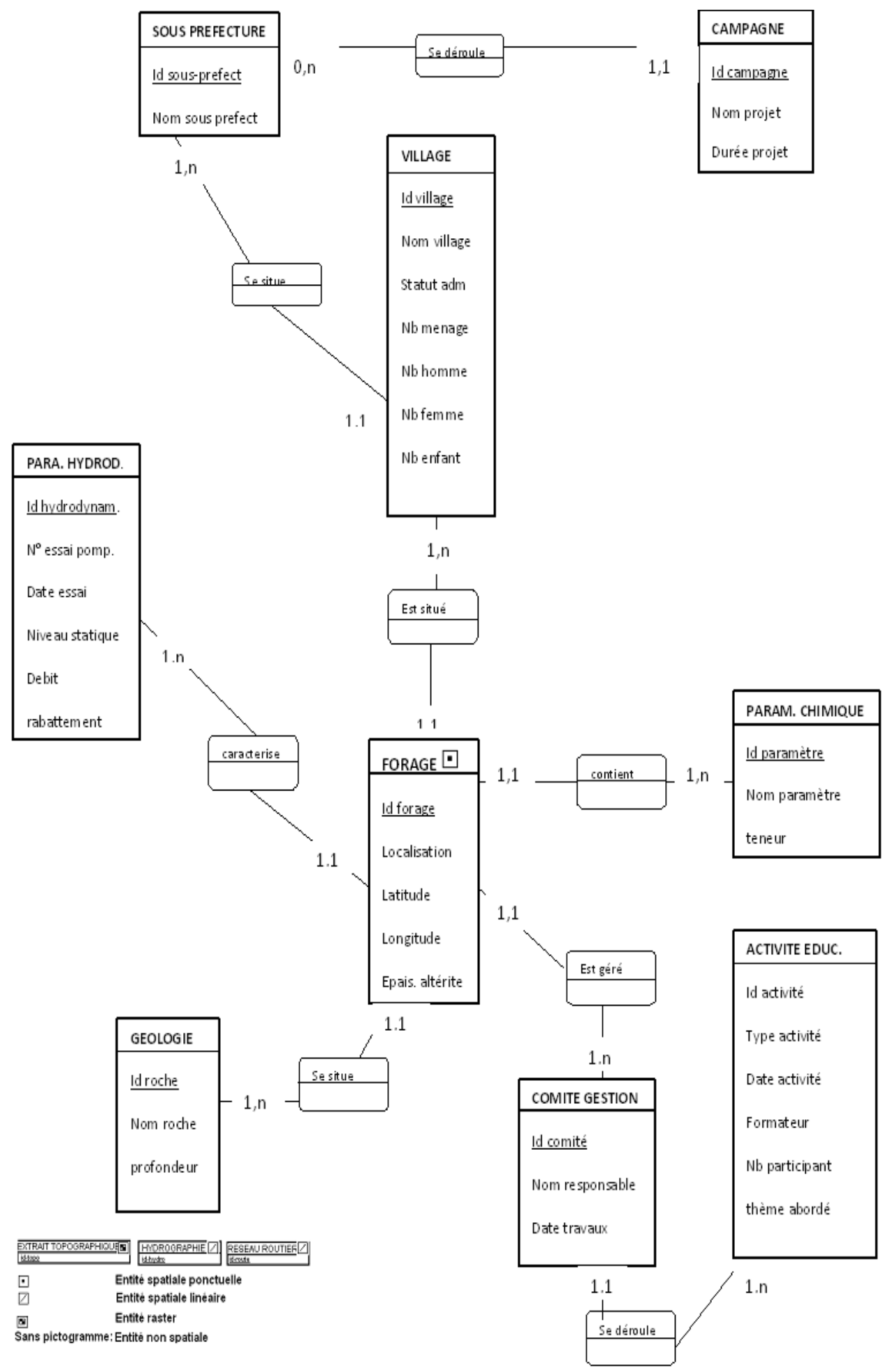

Figure 3 : Schéma du modèle conceptuel des données. 


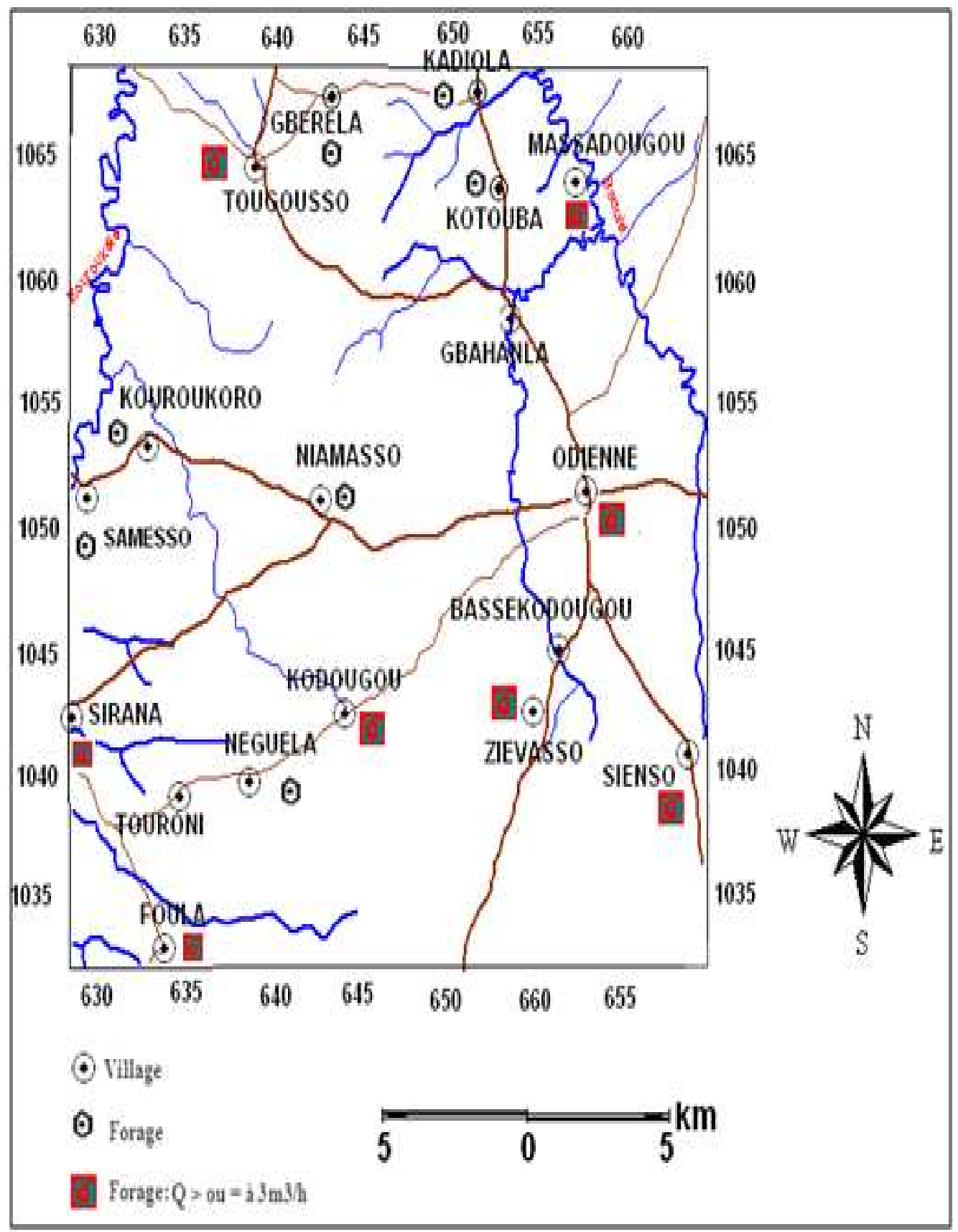

Figure 4 : Résultats de la requête : «Quels sont les forages qui ont un débit $\geq$ à $3 \mathrm{~m}^{3} / \mathrm{h}$ et dont l'épaisseur d'altérite est comprise entre 10 et $40 \mathrm{~m}$ ?». 


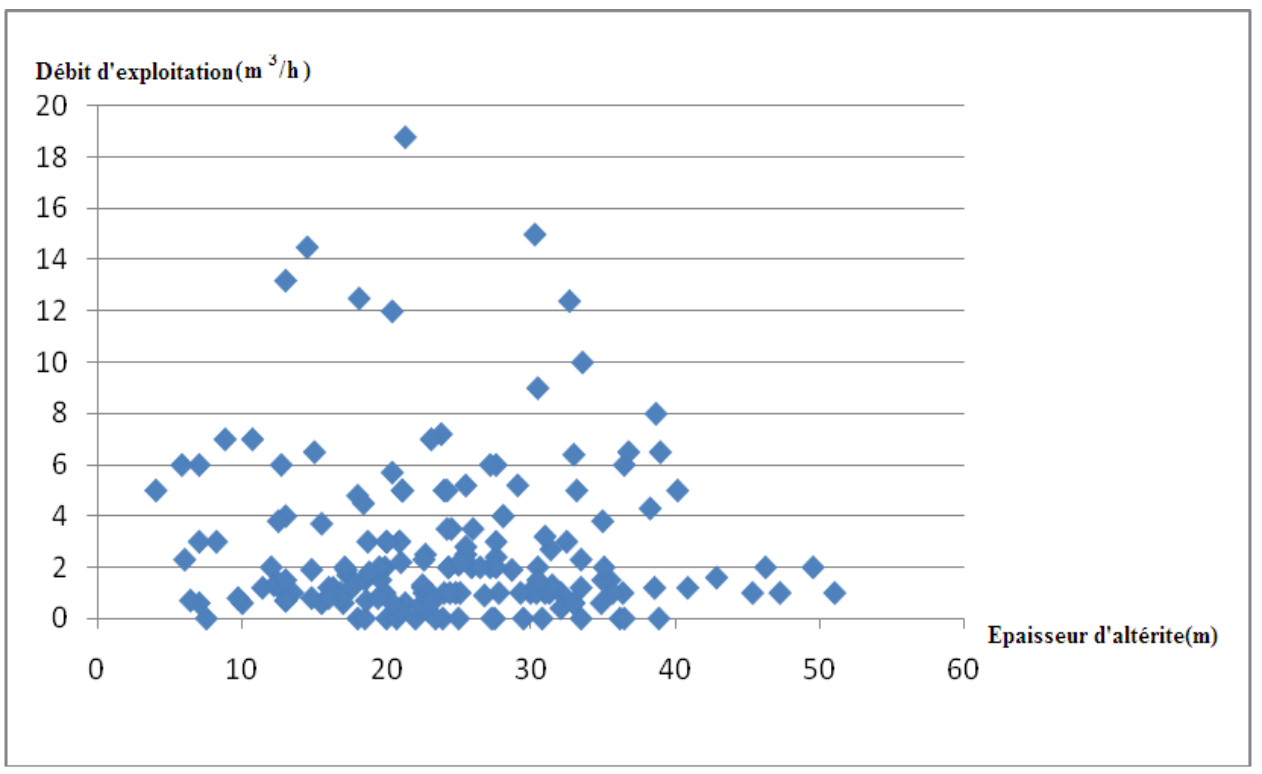

Figure 5: Variation du débit d'exploitation en fonction de l'épaisseur d'altérite.

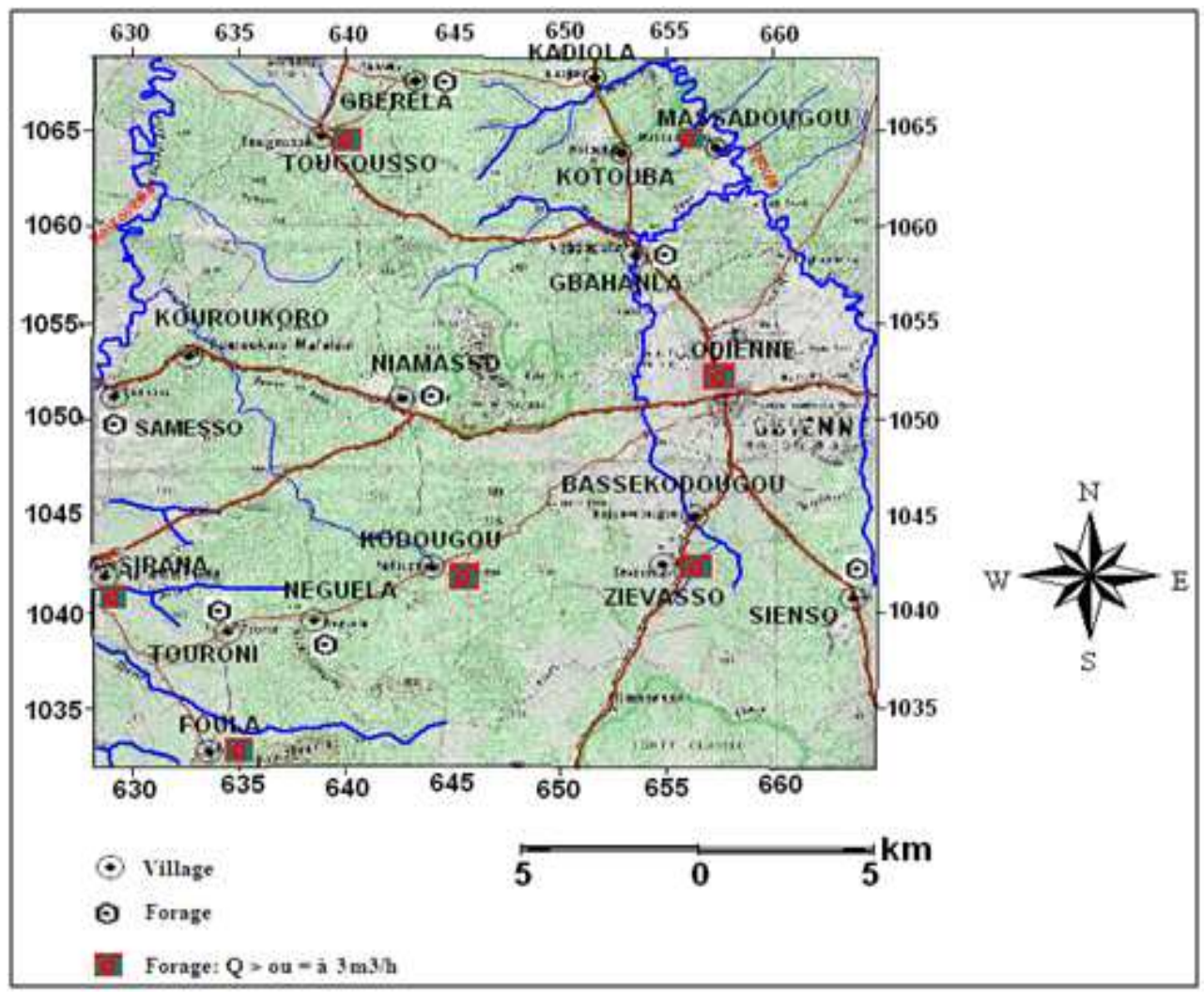

Figure 6 : Résultats de la requête : «Quels sont les forages qui ont un débit $\geq$ à $3 \mathrm{~m}^{3} / \mathrm{h}$ et dont la profondeur totale est comprise entre 40 et $70 \mathrm{~m}$ ?». 


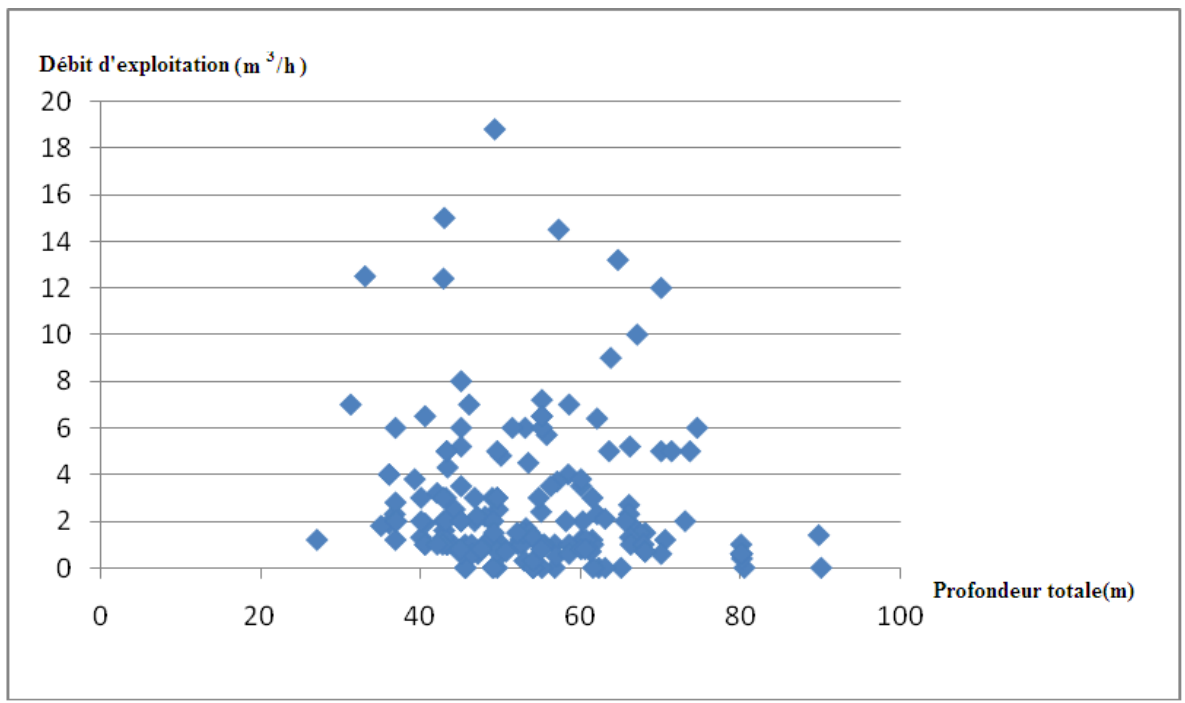

Figure 7: Variation du débit d'exploitation en fonction de la profondeur totale des Forages.

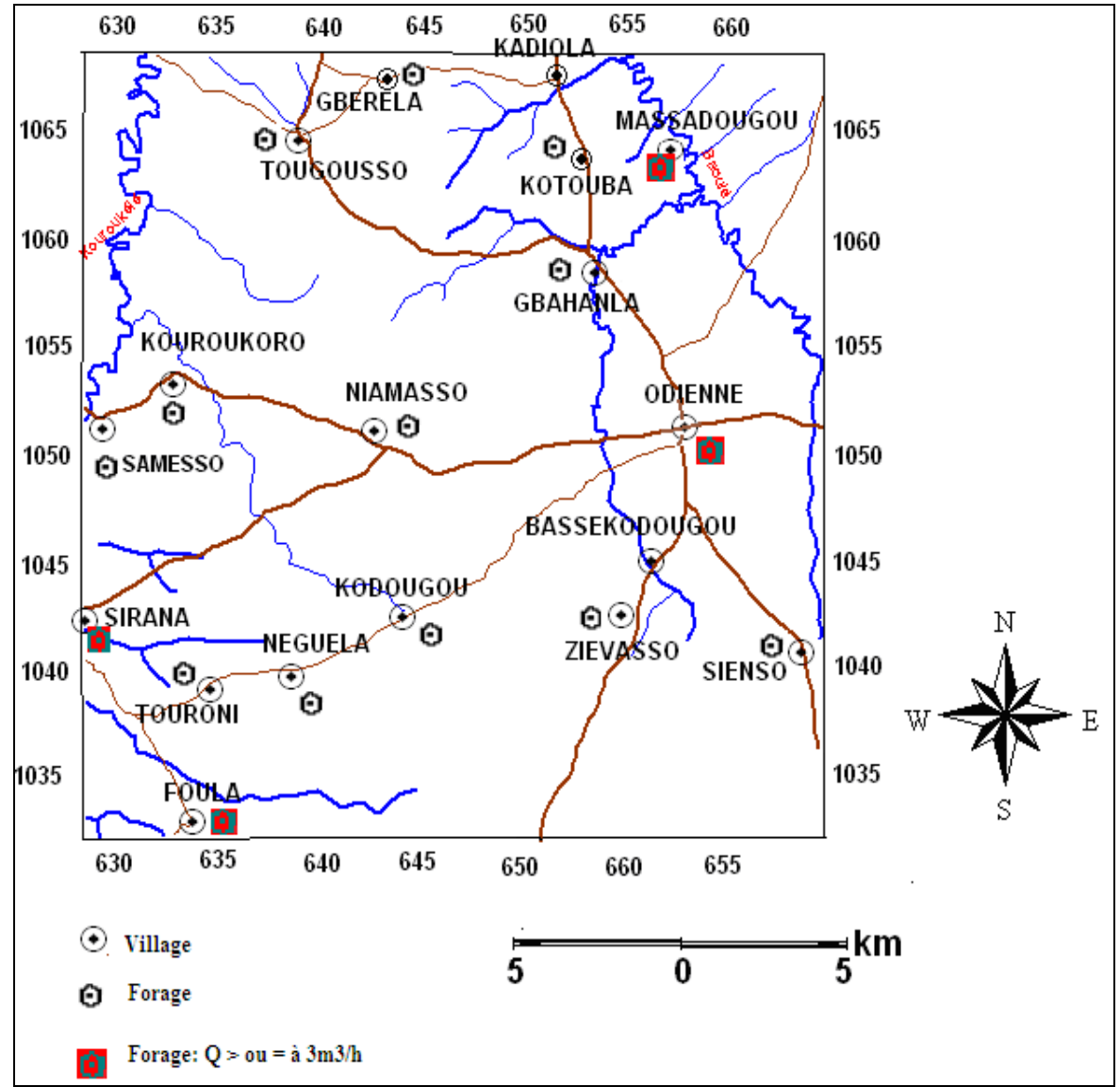

Figure 8 : Résultats de la requête : «Quels sont les formations géologiques dans lesquelles l'on rencontre des débits $\geq$ à $3 \mathrm{~m}^{3} / \mathrm{h}$ ? ». 
Tableau 1 : Localités ayant des forages qui ont un débit $\geq$ à $3 \mathrm{~m}^{3} / \mathrm{h}$ et dont l'épaisseur d'altérite est comprise entre 10 et $40 \mathrm{~m}$.

\begin{tabular}{lrr}
\hline nomvillage & débit $\left(\mathbf{m}^{\mathbf{3}} \mathbf{h}\right)$ & altérite $(\mathbf{m})$ \\
\hline TOUGOUSSO & 3,2 & 25,1 \\
MASSADOUGOU & 15 & 30,3 \\
ODIENNE & 7 & 23,1 \\
KODOUGOU & 3 & 27,6 \\
SIRANA & 3,6 & 27,6 \\
ZIEVASSO & 3 & 35,1 \\
FOULA & 6 & 12,7 \\
SIENSO & 3,1 & 25,5 \\
\hline
\end{tabular}

Tableau 2 : Localités ayant des forages qui ont un débit $\geq a ̀ ~ 3 \mathrm{~m}^{3} / \mathrm{h}$ et dont l'épaisseur d'altérite est comprise entre 40 et $70 \mathrm{~m}$.

\begin{tabular}{lrr}
\hline nomvillage & débit $\left(\mathbf{m}^{\mathbf{3}} \mathbf{h}\right)$ & profondeur totale $(\mathbf{m})$ \\
\hline TOUGOUSSO & 3,2 & 47 \\
MASSADOUGOU & 15 & 42,9 \\
ODIENNE & 7 & 41,2 \\
KODOUGOU & 3,1 & 46,7 \\
SIRANA & 3,2 & 46,7 \\
ZIEVASSO & 3 & 43,1 \\
FOULA & 6 & 51,4 \\
\hline
\end{tabular}

Tableau 3 : Formations géologiques dans lesquelles l'on rencontre des débits $\geq$ à $3 \mathrm{~m}^{3} / \mathrm{h}$.

\begin{tabular}{lrl}
\hline nomvillage & débit $\left(\mathbf{m}^{3} \mathbf{h}\right)$ & nom_roche \\
\hline MASSADOUGOU & 15 & gneiss \\
ODIENNE & 7 & granite à biotite \\
SIRANA & 3 & granite à biotite \\
FOULA & 6 & granite à biotite \\
\hline
\end{tabular}

\section{DISCUSSION}

L'élaboration de cette base de données pour la gestion des forages en milieu fissuré a aboutit à la mise en place d'un prototype de démonstration avec des cas précis pour le tester. Ainsi, l'implantation du prototype permet de voir si la structure de la base de données permet d'extraire les informations nécessaires à la réalisation des traitements requis dans le cadre de la problématique énoncée au début de cette étude. En second lieu, le prototype représente un exemple pratique de l'application des SIRS à la gestion des ressources en eau, domaine pour lequel la méthode MERISE n'a pas été spécifiquement développée. Le prototype constitue alors un moyen de dialogue pour continuer la réflexion et aboutir à la réalisation d'une interface graphique. 
Les analyses statistiques effectuées sur les bases de données de forages de la région du Denguelé à l'aide de cet outil, ont permis de montrer que, suivant la profondeur, les forages dont la profondeur est comprise entre 40 et $70 \mathrm{~m}$ sont les plus productifs. Suivant les formations géologiques, on constate que les débits $\left(\mathrm{Q} \geq 3 \mathrm{~m}^{3} / \mathrm{h}\right)$ s'observent aussi bien dans les granites à biotite que dans les gneiss. Toutefois, dans la majorité des cas, les débits d'exploitation des granites à biotite sont inférieurs à ceux des gneiss. Ces résultats qui confirment les travaux des études antérieures effectuées dans la région par Kouadio et al. (2009), indiquent que la profondeur optimale pour avoir un débit d'exploitation maximal pourrait se situer autour de $68 \mathrm{~m}$.

Notons que, pour Savané et al. (1997), une importante épaisseur des altérites peut devenir un facteur de productivité dans la réalimentation des aquifères fissurés, à condition que celles-ci présentent une bonne perméabilité. Dans le cas contraire, elles tendent à s'opposer à la réalimentation des fractures sous-jacentes. C'est ce qui expliquerait la tendance à la baisse des débits en fonction de l'épaisseur.

Par ailleurs, des travaux (Kouadio et al., 2008), ont montré que les granites à deux micas, les gneiss et les dolérites font parties des formations dites à forte densité de fracturation. On perçoit logiquement une relation de proportionnalité entre la densité de fracturation et la productivité des forages. Toutefois, selon Kouamé et al. (2005), en milieu de socle fissuré, les réservoirs sont discontinus et toutes les fractures ne sont pas hydrauliquement actives et donc productives. En somme, notons que la connaissance de ces informations est importante pour la planification des activités d'implantation des forages.

Le SIRS constitue donc un outil souple et performant d'analyse et d'aide à la décision, car il contribue à la collecte des informations directes, puis à la production d'informations dérivées (Joerin, 1995). Pour Thériault (1996), l'intégration de données à références spatiales donne une place importante au territoire par la possibilité de repérer la position des forages. Dans le cadre du prototype élaboré avec MapInfo, les traitements réalisés ont permis de tester et de valider les performances d'un tel outil.

\section{Conclusion}

L'utilisation du SIRS a permis d'élaborer un prototype pour l'exploitation des données de forages de la région du Denguelé. Ce prototype a été testé avec succès pour localiser et analyser les forages qui ont un débit $\geq$ à $3 \mathrm{~m}^{3} / \mathrm{h}$ et dont l'épaisseur d'altérite est comprise entre 10 et $40 \mathrm{~m}$, les forages qui ont un débit $\geq$ à $3 \mathrm{~m}^{3} / \mathrm{h}$ et dont la profondeur totale est comprise entre 40 et 70 $\mathrm{m}$ et les formations géologiques dans lesquelles l'on rencontre des débits $\geq$ à $3 \mathrm{~m}^{3} / \mathrm{h}$. Les résultats obtenus sous forme de tableaux, de graphique ou statistique, confirment que les systèmes d'information à référence spatiale (SIRS) constituent des excellents outils d'analyse permettant d'apporter une aide précieuse à la planification et au suivi des forages. Ainsi, cette base de données enrichie par une multitude de requêtes statistiques peut servir de guide à une nouvelle campagne de forages dans la région.

\section{REFERENCES}

Barazzuoli P, Bouzelboudjen M, Cucini S, Kiraly L, Menicori P. Salleoni M. 1999. Olocenic alluvial aquifer of River Cornia coastal plain (southern Tuscany, Italy): Database design for groundwater management. Environmental Geology, 39(2): 123-143.

Barbier P. 2002. Cours MapInfo V6.5 IGN ENSG CERIG.

Boker E. 2003. Développement d'une base de données hydrogéologiques liée aux forages en milieu fissuré et analyse geospatiale par système d'information géographique, certificat de géomatique $3^{\text {ème }}$ cycle, Université de Genève. p50.

Golay F. 1997. Systèmes d'information à référence spatiale I. Notes de cours SIRS 
à l'EPFL. Lausanne. Suisse.

INS. 2000. Recensement général de la population et de l'habitat 1998.Volume III : données socio-démographiques et économiques des localités. Tome 1 : résultats définitifs par localité, région du Denguelé.

INS. 2004. Situation démographique nationale, volume $\mathrm{V}$ : tome 4 , atlas des infrastructures socio-économiques de la région du Denguelé.

Joerin F. 1995. Méthode multicritère d'aide à la décision pour la recherche d'un site. Revue Internationale de Géomatique, 1(5): 37-51.

Kedaid FZ. 2006. Développement de la base de données géothermique de l'Algérie par un système d'information géographique, Revue des Energies Renouvelables, 9(4): $253-258$.

Kouadio KE, Savane I, Lasm T, Biemi J. 2008. Hydrogeology Prospecting in Crystalline and Metamorphic Area by Spatial Analysis of Productivity Potential European Journal of Scientific Research, 22(3): 373-390.

Kouadio KE, Soro N, Savane I. 2009. Stratégie d'optimisation de la profondeur des forages en contexte de socle: application à la région du Denguelé, Nord-Ouest de la côte d'ivoire. Revue des Sciences de l'Eau, 22(5): 1- 15.

Kouame KF, Akaffou AG, Lasm T, De Dreuzy JR, Davy P, Bour O. 2005. Simulation des écoulements dans les réservoirs fracturés: application au socle
Archéen de Touba (Nord-Ouest de la Côte d'Ivoire). Actes du Colloque Internationale SITIS 05, Yaoundé (Cameroun), du 27 Novembre au 1er Décembre 2005 : 39-46.

Lakhoua MN. 2007. Utilisation du SIG dans une entreprise industrielle pour l'analyse et la prise de décision, Schedae, Prépublication $n^{\circ} 47$, Fascicule $n^{\circ} 2$ : 309-314.

Lili Chabaane Z, Friaa I, Rhouma A, Ferchichi M. 2002. Choix d'un site de décharge de déchets industriels : Utilisation de SIG et de l'analyse multicritère, Proceedings of International Symposium on Environmental Pollution Control and Waste Management 7-10 January 2002, Tunis (EPCOWM'2002) : 425-436.

Pennober G, Odon O, Join JL, Folio JL. 2004. Approche par analyse spatiale de la faisabilité de captage d'eau souterraine sur le Massif du Piton de la Fournaise (île de La Réunion -France - Océan Indien), Cybergeo.

Savane I, Goze BB, Hugh Q, Gwyn J. 1997. Evaluation de la productivité des ouvrages dans le socle par l'étude des fractures et le GIS dans la région nordouest de la Côte d'Ivoire: Hard Rock Hydrosystems (Proceedings of Rabat Symposium S2, May 1997), IAHSPubl.no. 241, 1997 1UJ.

Theriault M. 1996. Systèmes d'information géographique - Concepts fondamentaux. Département de Géographie, Université Laval, Québec. 